\title{
ARGUMENTACIÓN Y ARGUMENTO
}

\section{Lázaro CARRILLO GUERRERO}

Universidad de Granada

lzro@msn.com

Resumen: Argumentar es interaccionar, estructurando textualmente unos argumentos con la fuerza ilocutiva de su discurso. Donde todo argumento requiere de una operación mental para llegar a unas conclusiones mediante la construcción textual que realizan el sistema gramatical y el sistema retórico.

Palabras clave: Discurso. Texto. Retórica. Realidad. Cognición.

Abstract: To argue is to interact, structuring some arguments in the text with the ilocutive force of its discourse. Where, every argument requires a mental operation to reach some conclusions through the textual construction which the grammatical system and the rhetoric system realize.

Key words: Discourse. Text. Rhetoric. Reality. Cognition. 


\section{INTRODUCCIÓN}

Argumentar es construir una realidad a través del lenguaje, mediante un proceso, el discurso, y un producto, el texto; pero dentro de la variabilidad que el uso de la lengua conlleva. En este marco, la lengua despliega una dimensión argumentativa, ya que su uso tiene un rol intencionado, y éste existe de acuerdo con las relaciones que se establecen entre los interlocutores. Entendemos, pues, que la argumentación está situada en una perspectiva sociolingüística, y ocupando una posición relevante en esas dos perspectivas complementarias que Halliday (1978: 10) llama intra-organism (los procesos cognitivos que están implicados en hablar y en entender), y inter-organism (el organismo humano en interacción con otros organismos humanos a través del uso de la lengua). Y de manera general, podemos decir que la actividad lingüística funciona argumentativamente en su dimensión comunicativa, la cual ha sido denominada indistintamente como discourse function, speech acts, illocution, etc. Pues, argumentar no solamente es convencer, hacer creer, descubrir lo verdadero, sino que también es influenciar: lograr o provocar reacciones en nuestros interlocutores.

Caron (1992), desde una perspectiva psicolingüística, considera al discurso como un proceso con una orientación argumentativa:

... a discourse always has a more or less argumentative function which is related not only to the content but also to the linguistic means employed (Caron, 1992: 165).

Y esta orientación argumentativa no solamente comunica una cierta cantidad de información que el receptor necesita estimar para reconstituir la incipiente representación; sino que, también, constituye una ruta o itinerario que, orientada de acuerdo a un propósito (goal-oriented), está ideada para obtener una cierta influencia en las creencias, actitudes y conducta del receptor (o auditorio), por medio de una representación que no solamente es enriquecida, sino que también es transformada y corregida en función de los objetivos del hablante (o de los interlocutores) (Caron, 1992: 165).

Como proceso comunicativo, la acción argumentativa es procesada en ese componente del sistema comunicativo humano, bien codificado, que Givón (1995: 395 y ss.) llama multi-propositional discourse. Este componente junto con otros dos, propositional information y conceptual lexicon, organizan de forma concéntrica el sistema de representación cognitiva, que junto con los sistemas de codificación (peripheral sensory-motor coding system, gram- 
matical coding system) combinan estos módulos funcionales, interaccionando mutuamente en la comunicación humana. Y donde habría que destacar, desde el horizonte argumentativo, dos aspectos que Givón apunta en el funcionamiento de estos componentes, citados anteriormente: a) la presunción que los miembros de la misma comunidad lingüística tienen de compartir culturalmente el mismo significado ${ }^{1}$; b) y el hecho de que las relaciones, cualidades, estados, etc., que la información proposicional transmite pueden pertenecer al mundo externo, al mundo interno mental, al mundo culturalmente mediatizado, o a varias combinaciones de estos mundos.

\section{NATURALEZA ARGUMENTATIVA}

Realmente, nosotros no comunicamos mediante la lengua, comunicamos mediante el discurso (Beaugrande, 1997). Nuestra conducta lingüística es algo más que lengua, es discurso. De modo que los actos o eventos comunicativos que realizamos, lo hacemos mediante el discurso. Y todo discurso implica un diálogo. Usar la lengua es establecer una relación de diálogo. Un diálogo entre la lengua y su contexto, y un diálogo constante entre la realidad y la realidad, a través del uso de la lengua. Un diálogo, donde el propósito fundamental del hablante es formular un mensaje para que funcione con efectividad como acto comunicativo. Ello lleva consigo que las características del auditorio, así como la percepción que el hablante tenga de éste y de la situación, puedan determinar algunos aspectos de la forma del mensaje. Y entendemos que es en este espacio retórico-argumentativo donde se logra la comunicación:

Communication is successful only when the hearer infers the speaker's intentions from the character of the utterance he produced (Fodor, 1979: 103).

En todo discurso hay retórica y en todo discurso hay argumentación. Entendemos que la retórica y la argumentación son cuestiones que abarcan la caracterización del discurso: ya que usar la lengua es comunicar e interaccionar con unas ideas, creencias y emociones en unas situaciones determinadas. Y discurso es esa interacción, con una concreción textual. De modo que, el discurso como proceso y el texto como producto tienen en común esa dimensión interaccional de naturaleza retórico-argumentativa ${ }^{2}$. Donde la va-

1 «Both 'same meaning' and 'same community' are somewhat elastic notions» (Givón, 1995: 446).

2 Ver Carrillo (2005). 
riabilidad y la adaptabilidad pueden considerarse como las grandes características comunicativas de nuestra conducta lingüística. Y el registro sistematiza estas características comunicativas actualizando la lengua de acuerdo con un proceso semántico-pragmático - el discurso- y en un producto gramático-retórico —el texto-. Por lo tanto, la argumentación no es un género determinado del discurso para unos usos específicos, u ocasionales. Sino que es un discurso que subyace en la base de todo acto de habla. Y en consecuencia, la argumentación está en la acción de todo discurso.

En cada uno de los diferentes enfoques al análisis del discurso hay un principio fundamental: considerar a la lengua como una interacción social. Lo cual tiene dos consecuencias importantes (Schiffrin, 1994: 415), que para nosotros sustentan el contexto argumentativo de todo uso de la lengua: 1) la interacción social es un proceso mediante el cual una persona, usando la lengua, obtiene un efecto en la otra, y 2) este proceso está implicado en un intercambio en el cual nuestras actividades son dirigidas hacia otras personas, y las actividades de los otros hacia nosotros.

Hay una respuesta común en los investigadores que estudian la argumentación ${ }^{3}$ : que ésta es un discurso que pone en juego unos actores sociales (Golder, 1996: 111). A través de ellos, el uso de la lengua constituye la expresión de un punto de vista sobre la realidad. Y ese punto de vista implica la propuesta de una acción ${ }^{4}$ : la acción de una fuerza ilocutiva que supone una interacción argumentativa ${ }^{5}$. Es decir, la acción de un propósito comunicativo o una intencionalidad que tiene una naturaleza argumentativa:

El propósito comunicativo, la intención del emisor, forma parte del concepto retórico de la convicción y la persuasión a través de la argumentación (Martínez-Dueñas, 2002: 9).

\footnotetext{
${ }^{3}$ Para un panorama completo acerca de los diferentes enfoques e investigaciones sobre la argumentación cf. Eemeren et al. (1996); y de una forma más abreviada ver (entre otros) Cox y Willard (1982) y Eemeren (2002).

${ }^{4}$ Entendemos que esta acción puede estar orientada hacia el convencimiento de uno mismo o hacia uno o más interlocutores.

5 Jacobs (1987: 236-7; en Lo Cascio, 1998: 243) aprecia en la tradición de los estudios norteamericanos dos tendencias para el análisis del discurso argumentativo: a) la argumentación como un procedimiento en el que un individuo llega a una conclusión, y b) la argumentación como una forma de llegar a un consenso entre la gente mediante la interacción. Nosotros entendemos que en las dos tendencias la argumentación supone una fuerza ilocutiva y una interacción.
} 
Desde esta misma perspectiva, Leith y Myerson (1989) resaltan la naturaleza retórica ${ }^{6}$ de la argumentación:

To argue is to engage with other views and voices, either explicitly (dialogue) or implicitly, anticipating how others may react to what one says. A second point is that argument is about address, about people addressing other people and other views (Leith y Myerson, 1989: 81).

Por su parte, Eemeren y Houtlosser (2000: 2) afirman:

...there is a rhetorical (pragmatic) aspect to all argumentative discourse: the participants are always aiming for the effects that suit them best.

Pero además, en toda esta naturaleza retórica de la argumentación, hay otro ingrediente: el deseo de compartir, que Vignaux (1988: 5) señala, y que es inherente a todo uso de la lengua. Este deseo de compartir, lo muestra, también, muy claramente el texto correspondiente a una situación comunicativa específica: una página ${ }^{7}$ de Internet (Defeat Depression), donde los usuarios envían cartas, por correo electrónico, sobre el tema de la depresión, con el deseo o la intención de, y escritas con la fuerza ilocutiva de: compartir las experiencias personales, servir de gran ayuda para quienes las vayan leyendo, y contribuir con todo ello a vencer la depresión.

El uso de la lengua como medio de representación y de comunicación es una realidad diaria que está sustentada por la necesidad humana de argumentar, la cual modela a la lengua en un sistema interaccional, dentro de un marco de comunicación y entendimiento. Esta acción de argumentar está en el más básico y neutral proceso de representación y comunicación. Un proceso donde siempre habrá, influyendo en las estructuras lingüísticas, una intencionalidad en las actitudes del emisor, una aceptabilidad en el auditorio y una situacionalidad en el escenario comunicativo. Y donde, de acuerdo con Halliday (1978: 89), la lengua es controlada por la estructura social, y la estructura social es mantenida y transmitida a través de la lengua.

La función esencial del sistema de la lengua, y por tanto de su gramática (Dik, 1997), es pragmática, es decir: la función de ser un instrumento de interacción interpersonal. Y la dimensión argumentativa se sitúa en las pro-

${ }^{6}$ «Here, 'Rhetorical' refers to an attitude towards what people do with language and what language does for people» (Leith y Myerson, 1989: 88).

7 http://www.depression.org.uk./interact/letters.php?purpose $=2 \&$ the_id $=26 \&$ curr_page $=4$. 
piedades interaccionales o de diálogo que todo acto comunicativo y toda codificación lingüística tiene. Y por consiguiente, donde el uso de la lengua, su proceso (el discurso) y su producto (el texto), tiene, ante todo, una naturaleza argumentativa. La cual, entendemos, que se realiza mediante: una negociación retórica del significado, una lógica construcción de la realidad y una dinámica argumentativa en la producción y recepción textual.

En definitiva, la argumentación obedece a una esquematización, a la vez cognitiva y lingüística, que opera con unas finalidades en cada discurso: orientar al otro hacia un sentido, hacia una cierta concepción del mundo. Y en todo este marco, la realización del significado es una actividad humana, que pretende, a través de la lengua y del discurso, entender al hombre y a la vida, y construir una realidad social. La cuestión está en saber si la realidad que nosotros percibimos (construida con nuestras experiencias individuales y conocimientos colectivos) es la verdadera.

\section{ACCIÓN ARGUMENTATIVA}

Perelman y Tyteca (1989) muestran que la argumentación es retórica ${ }^{8}$, así como Eemeren y Grootendorst (1984) señalan que la argumentación es un conjunto de actos del discurso9. Nosotros, entendemos que ambas afirmaciones tienen la misma base. La argumentación se construye sobre una base retórica ${ }^{10}$ : los caracteres y estados del orador (ethos) y su auditorio (pathos), estableciendo entre ellos una interacción pragmática. Pero, la argumentación es sobre todo retórica porque en el centro se sitúa el ser humano con su pensamiento, sus sentimientos y sus acciones. Y, por ejemplo, aunque es difícil

${ }^{8}$ Ellos parten del principio según el cual la argumentación existe porque hay un auditorio. Relativizando, así, el carácter lógico del discurso. Donde, frente al lenguaje formal de tipo lógico-matemático, exento de ambigüedad porque trata sólo de proposiciones verdaderas y evidentes, está el lenguaje natural donde las posibilidades son infinitas, hay una flexibilidad necesaria, y donde se administran las incertidumbres de la semántica (connotaciones...) y el carácter afectivo de las relaciones entre los interlocutores.

${ }^{9}$ Eemeren y Grootendorst (1984: 163-169) consideran que las dos partes (los interlocutores) implicadas en la argumentación lo hacen para resolver racionalmente un conflicto (o una disputa), y deben de respetar un código de conducta. Este código exige entenderse sobre unas reglas o normas objetivas o intersubjetivas acerca de la aceptabilidad de los argumentos, y sobre unas reglas lógicas que se puedan aplicar para evaluar los argumentos. Con ello, pretenden, más bien, una teoría normativa de la argumentación.

${ }^{10}$ Retórica y argumentación son dos conceptos unidos. En la época clásica y de Aristóteles eran términos sinónimos. En la época Renacentista y Barroca, ambas divergen, la retórica toma un carácter literario, centrándose sobre la expresión, la forma y la figuras, y la argumentación se identifica con los métodos deductivos y demostrativos. En la época contemporánea Perelman, Toulmin y otros reanudan la sinonimia entre retórica y argumentación. 
separar estos tres elementos (pensamiento, sentimiento y acción) en el uso de la lengua, una de las cartas (The need for better understanding) del texto correspondiente a la página de Internet mencionada anteriormente (Defeat Depression):

\section{THE NEED FOR BETTER UNDERSTANDING}

Depression is one of the commonest forms of illness and has become one of the most easily treatable disorders. Many people can find adequate relief in a primary care setting. It is unfortunate that all too often this disorder goes unrecognised and often patients are not able to communicate clearly their distress to their doctors. I welcome this website which I am sure will help bring about an increased understanding of these disorders - and with better communication between patient and doctor much needless distress can be eradicated.

Professsor Gerg Wilkinson.

puede dividirse en tres partes consecutivas que, además de suponer tres conclusiones argumentativas apoyadas en premisas implícitas, pueden representar (en cierto modo, y plausiblemente):

- El pensamiento: Depression is one of the commonest forms of illness and has become one of the most easily treatable disorders. ...

- El sentimiento: It is unfortunate that all too often this disorder goes unrecognised and often patients are not able to communicate clearly their distress to their doctors.

- Y la acción: I welcome this website which I am sure will help bring about an increased understanding of these disorders - and with better communication between patient and....

Moeschler (1985: 56-9) señala tres condiciones necesarias a la noción de «acto de argumentación»:

1. El carácter intencional, presentando intencionalmente un argumento relacionado a una conclusión, y de lo cual deriva un valor argumentativo.

2. El carácter convencional, asociado a las marcas argumentativas: marcas axiológicas (de orientación positiva o negativa), operadores argumentativos (dentro de la misma oración), conectores argumentativos (entre oraciones), y responsables de la actividad argumentativa. 
3. El aspecto institucional, imponiendo un conjunto de normas en el cuadro de la interacción, y obligando al interlocutor a sacar un determinado tipo de conclusión.

Pero, además, la argumentación es un proceso que implica: una interacción discursiva entre interlocutores, y la utilización y producción de un texto lingüístico. Este proceso podría ser definido como social, cognitivo y lingüístico. Pero, creemos que es ante todo un proceso retórico, basado en la lógica de lo probable ${ }^{11}$. Y un proceso caracterizado por su carácter de negociación del significado, y por una fuerza ilocutiva que pretende actuar en las representaciones del interlocutor. De modo que la retórica pertenece a la estructura íntima de la argumentación. Y esta estructura íntima puede distinguirse por los siguientes rasgos esenciales:

1. Se dirige a un auditorio que es más bien particular (un auditorio universal constituye más bien un ideal), con sus competencias y sus creencias intelectuales y afectivas.

2. Se expresa en lengua natural, escrita u oral, mediante unas reglas gramaticales y unos principios retóricos.

3. Está virtualmente en la lengua, y se construye y actualiza de forma interaccional y cooperativa, en un proceso ostensivo-inferencial, donde unos enunciados crean una relevancia para construir los enunciados siguientes.

4. Sus premisas se basan en lo probable. Y, al apoyarse sobre lo probable, ello puede comportar unos elementos demostrativos, en el sentido de necesarios.

5. Su progresión se realiza sin necesidad de una lógica formal (stricto sen$s u)^{12}$, ya que su organización depende de la situación y del auditorio.

6. Sus conclusiones no son apremiantes, y expresan, ante todo, un acuerdo entre interlocutores, o comportan tanto a quien las acepta como a quien las rehúsa.

\footnotetext{
11 Esta lógica de lo probable está instalada especialmente en la completa realidad de las situaciones comunicativas, y construyendo unas determinadas estructuras lingüísticas. Ésta opera en la dinámica del texto o del discurso, y a través de suposiciones o estimaciones aproximadas y probables sobre que tipo de información se procesa, de acuerdo con un cotexto y un contexto determinado.

${ }_{12}$ El argumento casi-lógico imita la transitividad lógica, y puede desarrollarse según una combinatoria algebraica.
} 
Pero la argumentación es una acción de la lengua y no simplemente de las ideas o los conceptos. Es la lengua, con sus recursos y peculiaridades estructurales (polisemia, ambigüedad, modalidad, etc.) y con sus meta-funciones (conceptual, interpersonal, textual), la que da fuerza y función a los argumentos. Y es el carácter probable, retórico, de la palabra quien permite que las estructuras lingüísticas de los interlocutores actúen de manera subjetiva y necesiten de la argumentación.

Además, una argumentación generalmente utiliza o tiene necesidad de varios argumentos. El argumento puede ser más o menos desarrollado según diversos procedimientos: - paráfrasis que intentan valorizar la acción argumentativa; - inserción de elementos que tienen valor de prueba: ejemplos, datos estadísticos, etc.; - y múltiples recursos más, dirigidos a apoyar la validez de una posición o punto de vista.

Anscombre y Ducrot $(1988)^{13}$, haciendo intervenir a la enunciación ${ }^{14}$ como base de la argumentación, y teniendo en cuenta más su base lingüística, conciben a la argumentación como un enunciado $\mathrm{E}_{1}$ (o un conjunto de enunciados) destinado a hacer admitir otro enunciado $\mathrm{E}_{2}$ (o un conjunto de enunciados). $Y$ para que esto pueda suceder no basta con que $\mathrm{E}_{1}$ dé razones para $\mathrm{E}_{2}$, sino que la estructura linguística de $\mathrm{E}_{1}$ debe de satisfacer ciertas condiciones para que pueda constituirse, en un discurso, un argumento para $\mathrm{E}_{2}$. La lengua presenta unas exigencias para esta relación, ya que según sus investigaciones, algunos enunciados $\mathrm{E}_{1}$, aún proporcionando las mejores razones del mundo para hacer admitir otros enunciados $\mathrm{E}_{2}$, son sin embargo incapaces, en un discurso, de servir de argumentos a favor de $\mathrm{E}_{2}$. Pero, es un rasgo constitutivo de muchos enunciados orientar al interlocutor hacia un cierto tipo de conclusión (por el hecho de que se excluye otro tipo de conclusión). Consideran que los posibles encadenamientos argumentativos en un discurso están ligados a la estructura lingüística de los enunciados y no únicamente a las informaciones que ellos llevan ${ }^{15}$. Estas posibilidades de enca-

\footnotetext{
${ }^{13}$ Anscombre y Ducrot (1988) analizan los principios de la argumentación en la lengua, en el mismo proceso de enunciación. Perelman y Tyteca (1989), y la mayor parte de los investigadores, lo analizan en el discurso. Pero entendemos que, aunque el punto de partida sea diferente, en ellos hay un marco común: la retórica.

${ }^{14}$ «El acto de enunciación es aquel que consiste en el hecho mismo de elegir un enunciado marcado por tales o tales actos específicos. Los encadenamientos argumentativos pueden estar fundados, bien de manera intrínseca, sobre uno de los actos específicos, bien de manera extrínseca, sobre el hecho de la enunciación» (Anscombre y Ducrot, 1988: 136; traducción nuestra).

${ }^{15}$ En esta noción, Anscombre y Ducrot (1988) basan su hipótesis de retórica integrada: según la cual todas las relaciones argumentativas entre los enunciados no son deducibles de su contenido informativo. Igualmente se ha hablado de una pragmática integrada (Moeschler, 1985: 74) que distingue claramente en-
} 
denamientos argumentativos son determinadas a través del acto de argumentar. Ellos hacen una distinción entre acto de argumentar y acto de inferir.

El acto de argumentar, presente, según ellos, en la mayor parte de los enunciados, es determinado por la estructura lingüística de estos enunciados, y donde la argumentación sería una explotación posible entre otras.

$\mathrm{El}$ acto de inferir es un acto de lengua realizado necesariamente por medio de un enunciado, donde su fundamento es un hecho, y no necesariamente un enunciado.

Por otro lado, Anscombre y Ducrot (1988: 116) también diferencian entre acto de argumentar y valor argumentativo. En un discurso, a los enunciados que pueden autorizar una conclusión, se les reconoce un cierto valor argumentativo (orientado por la presencia o no de operadores y conectores argumentativos), pero el acto argumentativo es realizado a partir del valor argumentativo que es dirigido hacia la conclusión. En este proceso actúa el acto de orientación argumentativa ${ }^{16}$, que consiste en dar, explícita o implícitamente, una orientación (intención) argumentativa a un enunciado. Aquí, entendemos que si el acto de argumentar es tal acto porque conduce a una conclusión, esta conclusión no tiene necesariamente que ser impuesta, sino que puede orientar el discurso hacia un tipo de conclusión y hacia la exclusión de otras:

Cuando nosotros hablamos de argumentación, nos referimos siempre a unos discursos que comportan, al menos, dos enunciados $E_{-}$y $E_{-}$, donde uno es dado par autorizar, justificar o imponer al otro; el primero es el argumento, el segundo la conclusión. [...] Puede, por otra parte, hacerse que el enunciado conclusión esté puramente implícito, pero que pueda ser, en este caso, reestablecido fácilmente (Anscombre y Ducrot, 1988: 163; traducción nuestra).

Así pues, según ellos, la argumentación se construye mediante un enunciado $\mathrm{E}_{1}$ (o un conjunto de enunciados), presentado como un argumento para autorizar a otro enunciado $\mathrm{E}_{2}$ explícito o implícito. Y constituye la realización de dos actos: la enunciación del argumento de una parte, y de otra, el

tre los hechos pragmáticos, que son relevantes a las propiedades internas de la lengua (convencionales), y los que son externos a la lengua (contextuales o conversacionales).

${ }^{16}$ Moeschler (1988: 67) matiza entre la orientación argumentativa y la conclusión: la orientación argumentativa impone al interlocutor un procedimiento interpretativo preciso: tal operador argumentativo o tal conector argumentativo da tal tipo de indicaciones sobre la orientación de los enunciados que el modifica o articula. La conclusión es el acto realizado por la presentación de un enunciado destinado a servir una cierta conclusión. 
acto de inferir realizado cuando se expresa o se sobreentiende la conclusión. Hay pues un fundamento de la argumentación sobre las posibilidades de inferencia, situándose la argumentación enteramente en el nivel del discurso, y la inferencia en las creencias relativas a la realidad ${ }^{17}$, a la manera en que los hechos se realizan. Cuando ellos afirman que la lengua es fundamentalmente argumentativa se refieren a un valor argumentativo, que no es un valor concluyente, sino que es básicamente una dirección de argumentación. Ello quiere decir, no que los enunciados siempre sirven para sacar conclusiones, sino que la significación de las frases comportan unas indicaciones en cuanto al valor argumentativo. Según ellos, la significación no es de naturaleza fundamentalmente veri-condicional, sino que tiene un valor de acción (un valor argumentativo) e inscrito en una dinámica discursiva que está constituida por un conjunto de relaciones argumentativas.

Estas relaciones argumentativas de naturaleza lingüística vienen dadas por las marcas argumentativas que son los operadores y conectores argumentativos, y por las reglas argumentativas permitiendo la actividad argumentativa que son los topoi ${ }^{18}$. Los topoi representan los trayectos argumentativos que se deben de adoptar para alcanzar una conclusión determinada. Y los operadores y conectores argumentativos no apremian la clase de conclusiones, sino los trayectos argumentativos que se permiten alcanzar; de este modo, ellos orientan argumentativamente a un enunciado.

De esta forma, la significación de una frase es el conjunto de topoi que ella autoriza a aplicar desde que es enunciada. Elegir, en una situación concreta, enunciar una frase en vez de otra, es elegir explotar ciertos topoi en detrimento de otros. Desde este punto de vista, significar es imponer, de cara a los hechos, la adopción de puntos de vista argumentativos.

De aquí podemos sacar dos conclusiones:

a) Que la argumentación es principalmente una construcción o estructuración lingüística. Esta construcción o estructuración conlleva o indica un valor argumentativo. Pero entendemos que va más allá del plan-

${ }^{17}$ La concepción tradicional de la retórica ve a la argumentación como un juego a partir del lenguaje, y no como un juego de lenguaje. La conclusión que se puede sacar de un enunciado o de una serie de enunciados, no es en virtud de una característica esencial (presente en la lengua), sino simplemente porque en ciertos contextos, las informaciones vehiculizadas (desde el nivel lengua) en un enunciado (o una serie de enunciados) permiten ciertas inferencias puramente locales.

18 Para Aristóteles, el desarrollo de todo discurso está regulado por un conjunto de principios generales, que el llama topoi, los lugares comunes, que se admiten en el seno de una comunidad más o menos amplia. 
teamiento de Anscombre y Ducrot (1988): encadenamiento de enunciados-argumentos y enunciados-conclusiones, apoyándose sobre el acto de argumentar. Ya que también construye y estructura los enunciados.

b) Que a esta construcción y estructuración lingüística va unida el proceso lingüístico-cognitivo de inferencia, realizado por medio de la lengua, pero fundamentado en una determinada construcción de la realidad.

\section{CAMPO ARGUMENTATIVO}

En investigaciones realizadas sobre la competencia argumentativa de niños preescolares, O'Keefe y Benoit (1982: 172-173) llegan a la conclusión de que la competencia argumentativa se adquiere como parte de la adquisición lingüística, ya que los niños empiezan a hablar y a argumentar simultáneamente. Y añaden que los niños antes de empezar a hablar, ya desarrollan la capacidad de indicar, de alguna manera, el desacuerdo. Estos estudios nos reiteran, una vez más, que la argumentación es una actividad lingüística, con una naturaleza social donde existen diferentes voces (reales o virtuales).

Ehninger (1970) define la argumentación como un proceso correctivo que tiene lugar entre los interlocutores. Y en este sentido, Eemeren et al. (1997: 209) ilustran cuatro rasgos centrales al concepto de argumentación:

1. Una estructura inferencial característica: proposiciones presentadas como pretensiones, y otras proposiciones (razones) presentadas como justificación y/o refutación de estas pretensiones.

2. Dos roles comunicativos: un protagonista que presenta una pretensión y un antagonista que duda de esa pretensión, la contradice, o de alguna forma retiene el asentimiento.

3. Los argumentos que están incrustados en actos y actividades.

4. Los argumentos que implican medios cuestionables de construir un asunto o una causa.

En nuestra concepción de argumentación tendríamos que expandir más el punto 2, y no relegar la argumentación sólo al desacuerdo o al no asentimiento, puede haber un acuerdo tácito y previo (e incluso genérico) y haber 
argumentación. Por acuerdo genérico entendemos el empleo de estructuras lingüísticas genéricas en encuentros sociales.

Por ejemplo, las cartas o las intervenciones discursivas que se originan en la situación comunicativa del texto correspondiente a la página de internet mencionada anteriormente (Defeat Depression), no proceden de una contradicción o de algún desacuerdo entre los interlocutores que intervienen, sino que, más bien, proceden del acuerdo previo implícito, e instrumentalizado por el interés o intención, de vencer a la depresión. Recordemos que esta situación comunicativa trata de una página de internet, donde los usuarios envían cartas, por correo electrónico, sobre el tema de la depresión, con el deseo de, y escritas con la fuerza ilocutiva de, compartir las experiencias personales, y de servir de gran ayuda para quienes las vayan leyendo. Y que, a su vez, la lectura de estas cartas y la identificación con su problemática motiva el envío de otra carta o intervención discursiva.

Por ejemplo, la estructura genérica Every problem has a solution, del siguiente texto ${ }^{19}$ :

[Conversación telefónica entre Jamey y Andrew, abogados y compañeros de trabajo en la misma empresa jurídica. Jamey está en el despacho de ellos, y Andrew está convaleciente en el hospital]

JAMEY (frenético): Andy?! This is a disaster! We can't find your revisions on the Kronos complaint!

ANDREW: Slow down, Jamey, for Christ's sake!

JAMEY: I went down to Word Processing to pick up the corrected copy, but they said you haven't delivered the corrections. I told them you've been working on it at home, and...

[Andrew se olvida de sus dolores físicos, dándole más importancia a este asunto]

ANDREW: I brought it in last night, around midnight. It's in my computer.

JAMEY: What did you file it under?

[Jamey reposa sus manos en el teclado de Andrew]

ANDREW: K-R-O-one.

[Jamey escribe el nombre del archivo]

19 De la película Philadelphia, dirigida por Jonathan Demne, 1993, Columbia/Tristar. 
ANDREW: Jamey, I don't have to mention, do I, that we're up against a statute of limitations on this complaint which runs out in... (mira su reloj) Seventy-five minutes.

[Jamey mira fijamente y asombrado al monitor]

JAMEY: It's not here, Andy.

[Andrew cierra los ojos. No puede creerlo]

ANDREW: You go down to Word Processing and tell those motherfuckers they better come up with that complaint, now! Or they are fucking dead! You tell them that comes from me!

[Andrew cuelga el teléfono en su base, golpeándolo. Hace una pausa, respira profundamente, y se dice a sí mismo]:

ANDREW: Every problem has a solution. Every problem... has... a... solution.

[Más calmado, Andrew comienza a ponerse su camisa]

ANDREW (CONT.): Every problem has a solution...

nos puede servir para ilustrar un acuerdo genérico entre interlocutores, que la utilizan como conclusión (de unas premisas anteriores) para afrontar una situación determinada.

En la perspectiva pragmático-dialéctica ${ }^{20}$ (Eemeren, 2002, 2003; Eemeren y Grootendorst, 1984, 1987, 1988, 1992; Eemeren et al., 1993; Eemeren et al., 1997; Jackson y Jacobs, 1980; Walton, 1989, 1995) se asume que el propósito de la argumentación es resolver la diferencia de opinión, implicando con ello que la oposición entre los roles argumentativos (protagonista-antagonista) es un rasgo característico del discurso argumentativo. Aquí, el argumento es definido como un medio de intentar resolver una diferencia de opinión, explorando la relativa justificación del punto de vista que concurre. Esta teoría presenta un modelo de discurso argumentativo en términos de procedimiento de discusión, donde ofrece reglas para la interacción argumentativa y unas precondiciones asociadas que tienen que ver con las habilidades, actitudes y poder de los interlocutores. Así, esta manera de entender la argumentación como una forma de reparar o resolver diferencias de opi-

${ }^{20}$ La combinación de estos términos «pragmático-dialéctica» indican que se trata de una teoría en la cual: a) la argumentación es interpretada como un «acto de habla» complejo, donde se combinan la teoría de los actos de habla y la teoría de la argumentación (pragmático-), b) y el proceso de argumentación es analizado en términos de una disputa entre un protagonista y un antagonista (-dialéctica).

Eemeren (2003) describe el desarrollo de la teoría pragma-dialéctica de la argumentación, junto con un breve panorama de los actuales proyectos de investigación. 
nión hace que ella sea entendida como una acción incrustada dentro de un contexto discursivo más amplio, y en términos de principios generales interaccionales (Eemeren et al. 1997:218-9). Este modelo de argumentación social puede definirse de la manera siguiente:

Argumentation is a social, intellectual, verbal activity serving to justify or refute an opinion, consisting of a constellation of statements and directed towards obtaining the approbation of an audience (Eemeren et al, 1987: 7) ${ }^{21}$.

Dentro de esta misma perspectiva, pragmático-dialéctica, Jackson y Jacobs (1980), Jacobs y Jackson (1982, 1989) 22 aplican el análisis del discurso a la descripción de la argumentación conversacional, y consideran a la argumentación (pág. 207) como una actividad linguiística en un sistema abstracto de reglas, las cuales definen a esta actividad lingüística como argumentación. Se trata de un juego lingüístico, de carácter social y cooperativo, con unos movimientos y contra-movimientos que corresponden a actos de habla y fuerzas ilocutivas. Así, la argumentación nace (pág. 158) como respuesta al desacuerdo (efectivo, virtual, potencial o proyectado) entre dos actos de habla, y es definida (pág. 215) como un procedimiento mediante el cual dos o más individuos llegan a un acuerdo.

El modelo de Toulmin (1958) se centra en la forma y contenido del discurso argumentativo. De acuerdo con él, un argumento puede ser descrito no tanto como una estructura lógica o silogística, sino como un movimiento entre los siguientes elementos:

1. Se tiene una pretensión (claim).

2. Se ofrecen unos hechos o datos para apoyarla (data/grounds).

3. Se conectan estos datos a la pretensión que se persigue mediante unas justificaciones (warrants).

${ }^{21}$ Esta obra, Handbook of Argumentation Theory (Eemeren et al., 1987) es una reimpresión de su predecesora, The Study of Argumentation (Eemeren et al, 1984). Y ambas son las predecesoras de otra obra posterior, Fundamentals of Argumentation Theory (Eemeren et al., 1996).

${ }^{22}$ Desde la perspectiva de los actos de habla, Jacobs y Jackson aplican el análisis del discurso a la descripción de la argumentación conversacional. Su objetivo es describir cómo se realiza el juego lingüístico de la argumentación en la conversación. Dentro de la perspectiva pragmático-dialéctica (en el ámbito norteamericano), ellos utilizan, para su estudio, diálogos auténticos, mientras que Eemeren y Grootendorst (y su grupo: la Escuela de Amsterdam) utilizan, más bien, ejemplos de enunciados inventados en situaciones monologales. 
4. Se muestran y respaldan los fundamentos de estas justificaciones, al menos implícitamente (backing).

5. Se utilizan unos modificadores modales apropiados para moderar la pretensión, y señalar el grado de certidumbre (modal qualifiers [some, many, most, etc]).

6. Y se consideran unas posibles refutaciones (rebuttals).

Se trata de una conexión conceptual que es aceptable para quienes encuentran el argumento convincente y sólido. Y considerando que los diferentes argumentos pueden exhibir una gran variedad de tipos de estos elementos (claim, data/grounds, warrants, backing, modal qualifiers, rebuttals).

De acuerdo con esta concepción, Toulmin et al. (1984) caracterizan a los argumentos según los campos de legislación, ciencia, administración, arte y ética, y sobre la base de qué cuestiones son argumentadas, y qué tipos de elementos (claims, data/grounds, warrants, backing, modal qualifiers, rebuttals) y conexiones se hacen. Según esto, entendemos que podríamos caracterizar a los campos argumentativos como una realización cognitiva y sociológica que conecta, a través del elemento field (inseparable de los otros elementos del registro: tenor, mode) el uso de la lengua y la estructura social e ideológica. De modo que podemos hacer referencia a campos argumentativos tan generales (o lugares) como la política, la sanidad, la educación, etc. Pero la realización argumentativa de ellos requerirá una concreción en los elementos (field, tenor, mode) de la situación comunicativa, y donde: Field refleja el contenido o tema de interés central a la situación argumentativa; Mode refleja la manera en que éste contenido está siendo comunicado; Tenor refleja la interacción que el hablante está teniendo con su auditorio, y cómo éstos (hablante y auditorio) tienen que ver de manera primordial con el proceso argumentativo que se desarrolla. Como por ejemplo en el texto anterior, donde el campo argumentativo se concreta en una cuestión puntual del quehacer laboral: la pérdida de un archivo de word. Y cuyo campo argumentativo viene caracterizado por field (pérdida de un archivo importante), tenor (Jamey y Andrew, compañeros de trabajo, aturdidos y preocupados) y mode (conversación oral por teléfono).

Toulmin (1958), además, al definir un argumento, fusiona y distingue el acto de argumentar, que puede ser considerado como un macro-argumento (a nivel del discurso), y los argumentos o micro-argumentos (a nivel de las oraciones) que lo componen: 
An argument is like an organism. It has both a gross, anatomical structure and a finer, as-it-were physiological one. When set out explicitly in all its detail, it may occupy a number of printed pages or take perhaps a quarter of an hour to deliver; and within this time or space one can distinguish the main phases marking the progress of the argument from the initial statement of an unsettled problem to the final presentation of a conclusion. These main phases will each of them occupy some minutes or paragraphs, and represent the chief anatomical units of the argument -its 'organs', so to speak. But within each paragraph, when one gets down to the level of individual sentences, a finer structure can be recognised, and this is the structure with which logicians have mainly concerned themselves. It is at this physiological level that the idea of logical form has been introduced, and here that the validity of our arguments has ultimately to be established or refuted (Toulmin, 1958: 94).

Nosotros, basándonos en esta definición construimos el esquema de la figura que viene a continuación, donde la argumentación o acto de argumentar puede ser tratado como unos macro-argumentos discursivos y unos micro-argumentos textuales, habiendo entre ellos una relación de interdependencia y correspondencia, en consonancia con el carácter explícito e implícito de sus premisas, y de acuerdo con la situación retórica en la que se ubican. Queremos subrayar que con los términos macro/micro-argumentos ${ }^{23}$ pretendemos diferenciar e identificar la argumentación en el discurso (proceso de la situación comunicativa) y, esa misma argumentación, en el texto (producto, con unas estructuras lingüísticas), con el matiz diferenciador de que las premisas explícitas se plasman en el producto (en el texto), y las premisas implícitas están situadas en el marco de su discurso. Así, la relación entre macro-argumentos y micro-argumentos es la relación entre lo explícito y lo implícito. Es una relación que define a la argumentación como discurso (proceso) y como texto (producto). Conduciendo todo ello a un proceso os-

${ }^{23}$ Nos gustaría evitar la interpretación e identificación de estos términos con otros que puedan ser susceptibles de ello. Como por ejemplo: con la noción de micro-estructura de un argumento, sobre la que se concentra la lógica deductiva. O con los siguientes conceptos distinguidos por Eemeren y Grootendorst (1992: 77): coordinatively compound argumentation (todos los argumentos se relacionan directamente con el punto de vista), subordinatively compound argumentation (el primer argumento se relaciona directamente al punto de vista, el segundo argumento al primero, que sirve como punto de vista, y así sucesivamente). O los distinguidos por Lo Cascio (1998: 137-9): argumentación múltiple (sumas de argumentaciones simples que conducen a la misma conclusión), y argumentación arracimada (argumentación múltiple subordinada).

Sí tienen cierta identificación con las nociones de macro-estructura y micro-estructura en el procesamiento del texto (Kintsch y Dijk, 1978; Dijk, 1980; Dijk y Kintsch, 1983). En concreto con la noción de micro-estructura de un texto, la cual está constituida por las proposiciones que están explícitamente presentes y aquellas que tienen que ser inferidas para asegurar la coherencia, formando todo ello el «texto base». 
tensivo-inferencial determinado, donde las estructuras y referencias lingüísticas no solamente reflejan lo explícito, sino que también llevan una carga importante de lo implícito, sobre lo cual tenemos que inferir. Y jugando ambos procedimientos (lo explícito y lo implícito) una dinámica crucial en la interacción argumentativa.

\section{DISCURSO: Macro-argumentos}

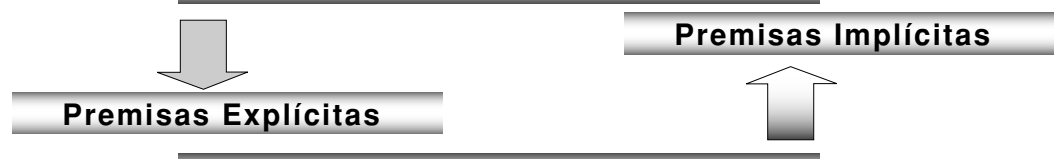

TEXTO: Micro-argumentos

Argumentación o acto de argumentar

El siguiente texto ${ }^{24}$, el discurso de Charles Spencer en el funeral de su hermana, la Princesa Diana, el 6 de eptiembre de 1997:

The Earl Spencer (Standard British English):

There is a temptation to rush to canonise your memory, there is no need to do so. You stand tall enough as a human being of unique qualities not to need to be seen as a saint. Indeed, to sanctify your memory would be to miss out on the very core of your being, your wonderfully mischievous sense of humour with a laugh that bent you double. Your joy for life, transmitted wherever you took your smile, and the sparkle in those unforgettable eyes. Your boundless energy, which you could barely contain. But your greatest gift was your intuition and it was a gift you used wisely. This is what underpinned all your other wonderful attributes and if we look to analyse what it was about you that had such a wide appeal we find it in your instinctive feel for what was really important in all our lives. Without your God-given sensitivity, we would be immersed in greater ignorance at the anguish of Aids and HIV sufferers, the plights of the homeless, the isolation of lepers, the random destruction of landmines.

Diana explained to me once that it was her innermost feelings of suffering that made it possible for her to connect with her constituency of the rejected. And here we come to another truth about her. For all the status, the glamour, the applause, Diana remained throughout a very insecure person at heart, al-

\footnotetext{
24 Speak Up, 1998, n. ${ }^{\circ}$ 148, 25-6. Barcelona: RBA Revistas SA.
} 
most child-like in her desire to do good for others so she could release herself from deep feelings of unworthiness of which her eating disorders were merely a symptom. The world sensed this part of her character and cherished her for her vulnerability whilst admiring her for her honesty.

constituye un discurso, donde, como en todos los discursos, hay un punto de partida integrado, implícitamente, por todas las presunciones y premisas que el hablante y el auditorio comparten y que conforman el conocimiento compartido (shared knowledge ${ }^{25}$ ) acerca de la vida de la Princesa Diana, y de la situación comunicativa de su funeral. Este punto de partida se sitúa en los macro-argumentos discursivos, desde donde se produce un texto o unos micro-argumentos. Pero, estos micro-argumentos están en una interacción constante con lo implícito de su discurso. Interacción desde donde el proceso ostensivo-inferencial conduce a la relevancia comunicativa. Por ejemplo, el texto consta de dos párrafos conduciendo, macro-argumentativamente, cada uno, a dos relevantes conclusiones implícitas:

1. En el primer párrafo, la conclusión -You stand tall enough as a human being of unique qualities not to need to be seen as a saint- se apoya textualmente en las premisas que se enumeran acerca de su vida (...your wonderfully mischievous sense of humour... your joy for life,... your God-given sensitivity,...), pero todo ello conduce a una conclusión relevante e implícita: she was a person full of life and full of goodness.

2. En el párrafo siguiente, los micro-argumentos textuales que se expresan (...her innermost feelings of suffering... a very insecure person at heart,...) conducen a otra conclusión relevante e implícita: she was a sensitive person with inner feelings of suffering.

Estas dos conclusiones implícitas sirven para apoyar — también implícitamente-, en la macro-argumentación discursiva, a la conclusión inicial: You stand tall enough as a human being of unique qualities not to need to be seen as a saint.

25 «... 'information' that discourse participants actually possess in common, or assume they possess in common ...» (Edwards, 1997: 137). 


\section{ARGUMENTAR}

Argumentar es interaccionar o comunicar. De modo que, la argumentación está en toda realización de la lengua, en todo acto comunicativo ${ }^{26}$. Pero, en este acto comunicativo se construye un texto que estructura los argumentos de una forma determinada. El texto argumentativo consiste en desarrollar unos argumentos a partir de una(s) premisa(s), y orientados hacia una conclusión. Los argumentos se entienden que son unas razones o unas significaciones que toman forma, a través de la lengua, en una situación discursiva, y que realizan movimientos de apoyo, de progresión, y de inferencia en un discurso (macro-argumentos). Y todo ello se realiza a través de unas estructuras textuales explícitas e implícitas, que conformarían los microargumentos textuales. Éstos tendrían la función que Dijk (1977:245) llama macro-performative ${ }^{27}$, es decir, ellos expresan la fuerza ilocutiva de todo su discurso.

Y esto lo podemos ver muy claramente en el siguiente texto ${ }^{28}$ :

\section{LOOK GOOD. FEEL BETTER. BE YOUR BEST.}

No matter what your body type, this is the best workout video for you.

donde de forma global, y micro-argumentativamente, se está diciendo algo más, y orientando con ello a una conclusión [ $\rightarrow$ so, buy this video and feel better doing its instructions and advices], formando parte, todo ello, de una macro-argumentación discursiva. De modo que, podemos afirmar que el

${ }^{26}$ En contraste con nuestro planteamiento, nos parece oportuno citar una afirmación que puede representar una posición crítica frente a la nuestra: «One danger associated with some recent work on argument is that the term argument itself becomes so broad that it loses all meaning. If argument is defined to include all disagreement, all comparison of construct systems, and all instances in which an individual believes that he or she is arguing then essentially all communication is argument» (Rowland, 1987: 155). Sin embargo, nuestra posición coincide con otras, como ésta de Moeschler (1985), la cual nos parece relevante. Él (pág. 14) parte del principio de que toda interacción verbal, donde el lugar de realización es la conversación, define un cuadro de coacción y de argumentación. Se trata de un espacio donde hay unas acciones y unas conclusiones involucradas, y donde los interlocutores están obligados a debatir, marcar unos puntos, negociar para llegar a una solución, confirmar opiniones o polemizar. Para Moeschler (1985: 72) la argumentación es indisociable de la enunciación. Queriendo decir con ello, que las propiedades de la actividad enunciativa son constitutivas de la actividad argumentativa.

27 «At the beginning of a longer speech we may say «I'll give you some good advice:», or at the end: «This is a promise». Such expressions are what we might call MACRO-PERFORMATIVES: the sentences themselves are not performative, but they express the illocutionary force of the discourse as a whole» (Dijk, 1977: 245).

${ }^{28}$ Extraído de la página de una revista, donde junto a la imagen de una top model (Elle Macpherson), se hace publicidad de la venta de un vídeo para hacer ejercicios físicos, titulado: Your Personal Best. 
discurso (macro-argumentos) y su estructura textual (micro-argumentos) son actos de habla globales (conformando un acto de habla global), y con unas pretensiones argumentativas en relación con los aspectos relevantes de la situación comunicativa.

Desde esta posición anterior (la de las pretensiones de los hablantes, y la de la situación comunicativa), entendemos que Willard (1989: 53) señala la relevancia de la situación comunicativa, y la relevancia de las posiciones de los interlocutores, al elaborar la siguiente definición de argumento:

An argument is a social encounter built upon the following minima: I assume that we disagree; I assume that you assume we disagree; I assume that I am arguing and that you agree that I am arguing; you assume that you are arguing and that I would agree that you are arguing (Willard, 1989: 53).

Estas dos relevancias señalan los dos marcos importantes con los que Willard (1989) identifica la argumentación: la comunicación y la interacción.

El acto de argumentar ha sido definido por Eemeren et al. (1984: 10-34) y Eemeren y Grootendorst (1984: 78-93) como un acto de habla complejo compuesto de una o varias proposiciones, que son los argumentos o las premisas, para apoyar otra u otras proposiciones, que son las conclusiones (o aquello que se pretende). Entre estos argumentos que componen el acto de argumentar, ellos distinguen entre pro-argumentos (para apoyar o justificar) y contra-argumentos (para rehusar o atacar), que muy posiblemente, en el transcurso del acto de argumentar, deriven en otros pro-argumentos y contra-argumentos. Ello hace que la argumentación sea un proceso complejo de diferentes tipos de argumentos.

Así, la argumentación se caracteriza por unas razones o significaciones que apoyan una afirmación o una tesis. Es decir, por unos tipos de argumento ${ }^{29}$ que el discurso va avanzando, en el texto, de acuerdo con el pensamiento e interacción de los interlocutores, y de acuerdo con la naturaleza y el encadenamiento de las estructuras. Un argumento es un acto retórico, y su fuerza viene dada por la dimensión retórica de su acto de argumentar. Si Ha-

${ }^{29}$ La tradición retórica clásica reconoce tres tipos de argumentos o géneros: judicial (son argumentos que establecen la verdad o falsedad de las alegaciones sobre la conducta de la gente y la exactitud de sus juicios acerca de su conducta; y cuyo rasgo principal es el acusar y el defender), deliberativo (argumentos que establecen la conveniencia de tomar o no acciones determinadas; y cuyos rasgo principal es el aconsejar y el desaconsejar) y epidíctico (argumentos que demuestran que alguien merece honor y alabanza; y cuyo rasgo principal es la alabanza y la crítica). 
bermas (1981) y Willard (1983), por ejemplo, plantean que la fuerza de un argumento es una cuestión epistémica ${ }^{30}$, lo epistémico, a nuestro entender, indica necesidad factual (basada en los hechos), probabilidad, posibilidad, etc., es decir, lo epistémico tiene un carácter retórico.

Partiendo de que todo argumento lleva consigo una fuerza retórica, podemos distinguir un tipo de argumento, cuya dimensión retórica queda aislada de él. Se trataría de unas premisas verdaderas, apoyando, mediante una relación deductivamente válida, a una conclusión, y todo ello aislado de una situación concreta o algún contexto determinado. Es decir, una relación lógico-formal en una predicación seriada, vacía de factores sociales, actitudes, creencias, modos de expresión, etc.

En contraste, Govier (2001: 81-86) expone tres ejemplos de sostenimiento específico para las premisas, que, a nuestro entender, son ejemplos de un sostenimiento retórico:

1. «Todo el mundo dice eso». Esta premisa afirma algo conocido prácticamente por todos, y formando parte de las creencias generales, y del contexto compartido por el argumento. Es un concepto casi normativo.

2. «Testimonio fiable». La premisa concierne con el testimonio de una persona que es fiable y digno de fe para el autor del argumento.

3. «Autoridad legítima». La premisa está apoyada por la autoridad directa de la persona que argumenta (o la autoridad indirecta de otra persona). En este caso, el argumentador posee unas competencias especializadas sobre el tema, y es reconocido como un experto.

Creemos, además, que estos tres tipos de sostenimiento circunscriben la argumentación a una comunidad determinada con unas creencias (e ideología) comunes y específicas.

Al igual que la lengua, como un sistema virtual de opciones disponibles para usar, puede distinguirse del texto, como un sistema actual en el que se han utilizado unas opciones en unas estructuras determinadas (Beaugrande y Dressler, 1981: 35); también podría hablarse de una argumentación virtual, donde la estructura gramatical de la lengua distingue entre argumento posible y argumento decisivo (Anscombre y Ducrot, 1988: 31). De modo que, podríamos considerar al argumento como una operación mental para llegar a

\footnotetext{
30 Esta palabra es de origen griego, significando conocimiento, entendimiento.
} 
unas conclusiones, definiciones, etc., mediante la construcción textual que realizan el sistema gramatical y el sistema retórico (un sistema de funciones para lograr a través del uso de la lengua unos propósitos comunicativos). Y desde esta perspectiva, un argumento puede definirse, en extensión, de la misma forma que se hace con un texto, ya que puede variar desde una palabra hasta varios volúmenes. Por ejemplo, el texto anterior (relativo al discurso de Charles Spencer en el funeral de su hermana, la Princesa Diana, el 6 de Septiembre de 1997), con sus dos párrafos enteros, puede ser tomado en su totalidad como un argumento con las dos conclusiones implícitas que hemos expuesto: she was a person full of life and full of goodness / she was a sensitive person with inner feelings of suffering. Pero en contraste, en el texto siguiente ${ }^{31}$ :

[Ben está tendido en la cama de su habitación, es verano, y acaba de graduarse]

MR. BRADDOCK'S VOICE: What is it, Ben?

BEN: I' $m$ just -

MR. BRADDOCK: —worried?

BEN: Well -

MR. BRADDOCK: About what?

$B E N$ : I guess - about my future

MR. BRADDOCK: What about it?

$B E N$ : I don't know. I want it to be-

MR. BRADDOCK: To be what?

$B E N$ (tranquilamente): Different

la palabra Different puede ser tomada como un argumento, textualmente situada en la interacción entre Ben y Mr. Braddock:

BEN: I don't know. I want it to be-

MR. BRADDOCK: To be what?

$B E N$ (tranquilamente): Different.

y discursivamente situada en premisas implícitas consideradas por Ben, acerca de su futuro (...about my future).

31 De la película The Graduate, dirigida por Mike Nichols, 1967, MGM Home Entertainment. 
Golder (1996:55-6) considera como argumento a todo segmento que apoya a otro segmento, sea por una relación de causalidad, de finalidad, de ejemplificación, de restricción, etc., pero con la condición de que el segmento que da apoyo no sea una reformulación del mismo nivel que el segmento apoyado. Y el segmento apoyado (la tesis o conclusión) puede a su vez apoyar a otro elemento y, por consiguiente, tener función de argumento.

Fahnestock y Secor (1982:20-2) ${ }^{32}$ caracterizan un argumento con tres elementos:

1. la afirmación de una tesis (cualificada, elaborada, complicada, o esparcida por todo el discurso), de acuerdo con un propósito, y caracterizada por la probabilidad ${ }^{33}$,

2. un auditorio, sobre el que se quiere tener algún efecto (hacer creer, aumentar su creencia, instar a actuar o a creer), y que va a influir sobre la forma de argumentar (fríamente o con pasión, con tanteo o con una fuerte convicción, elípticamente o en gran detalle),

3. unos fundamentos, razones, o premisas que apoyen la tesis, pero que dentro de ellos haya algunos supuestos (un supuesto o presunción es una premisa que no es dicha o escrita) que conformen las creencias y preconcepciones del campo común entre argumentador y auditorio.

De estos tres elementos, el más variable es el auditorio.

Además, Fahnestock y Secor (1982: ix) dividen los argumentos en dos clases:

a) Los que toman la forma de una proposición categórica, vinculando la evidencia a la definición, y cuyo tema que trata tiene unas características determinadas de pertenencia a un grupo.

b) Los que toman la forma de una afirmación sobre causas o efectos de un tema, proposición causal, enlazando dos acontecimientos por la acción de uno sobre el otro.

${ }^{32}$ Fahnestock y Secor (1982) entienden la noción de argumento en el mismo sentido en el que Aristóteles y Cicerón lo concibieron.

${ }^{33}$ Fahnestock y Secor (1982: 20) hacen la siguiente afirmación: «A thesis statement, a claim, a proposition to be supported, which deals with a matter of probability, not a fact or a matter of taste.» Sin embargo nosotros creemos que la afirmación sobre un hecho o sobre una cuestión de gusto, pueden caracterizarse también por la probabilidad, en cuanto que no van a manifestar una objetividad completa. Por ello, preferimos expresar a secas: «caracterizada por la probabilidad». 
Una evaluación puede ser uno de los dos o ambos. Sin embargo, una propuesta es una afirmación acerca de alguna acción que debería de ser realizada, y es una forma especial de declaración causal en la que se predice que ciertas acciones recomendadas mejorarían el estado actual.

Una proposición categórica (término prestado de la lógica) establece una relación entre su sujeto y su predicado, y toma la forma de una conclusión o definición, ya que implica un proceso concluyente, y se apoya en un proceso de definición para declarar acerca de la existencia o naturaleza de una realidad determinada. En el campo de la proposición categórica, están la comparación $^{34}$, y la disyunción, que son unas formas de argumentar, declarando acerca de la existencia o naturaleza de una realidad determinada, mediante el proceso de contrastar diferencias o semejanzas en el caso de la comparación, y mediante un proceso lógico de dividir una serie de posibilidades en dos alternativas en desavenencia en el caso de la disyunción ${ }^{35}$.

Una proposición causal establece una relación entre causa y efecto (ambos en una relación de influencia: primero la causa y, en consecuencia, el efecto) que es estructurada de acuerdo a lo que se pretende en el auditorio. Fahnestock y Secor (1982:254 y sigs.) clasifican tres tipos de proposiciones causales:

1. Algunas proposiciones con verbos causales indican claramente la causalidad y también el grado de ella (causalidad débil: improve, affect, take away from, etc., causalidad fuerte: destroy, create, trigger, etc.).

2. Muchas declaraciones apoyadas por argumentos causales pueden parecer proposiciones categóricas. En realidad, las razones para establecer la relación sujeto-predicado son las causas para construir la proposición categórica. Estas proposiciones pueden ser ambas cosas: declaraciones causales, y proposiciones categóricas (Prostitution is a victimless crime).

3. Declaraciones causales if-then (If $X$ then $Y$ ). Pero pueden ser expresadas en proposiciones causales directas ( $X$ causes $Y$ ). Algunas de ellas pueden ser una antecedente seguido por un consecuente, en vez de

34 «Some philosophers argue that the act of comparing is a more fundamental operation of the mind than that of defining» (Fahnestock y Secor, 1982: 163).

35 «An imperfect disjunction is best seen as a rhetorical device, a way of expressing something that helps emphasize it and shape the pattern of a reader's thinking. Setting up a disjunction forces a reader to think in terms of a limited set of alternatives» (Ibíd.: 178). 
causa seguido de efecto (If it is day (antecedente) now, then it will be night (consecuente) soon).

4. Declaraciones sobre un hecho ${ }^{36}$ añadiendo una razón o explicación (una causa) para ello (Dinosaurs are extinct because a supernova exploded 60 million years ago).

5. Predicciones racionales apoyadas sólo por cuidadosos argumentos causales ${ }^{37}$ (utilizando una analogía causal: if $A$ produced $B$ in the past; now with $A$, we can predict that $B$ will -is going to-follow).

Las proposiciones de evaluación y propuesta requieren de los componentes básicos que integran los argumentos causales y de proposiciones categóricas (Fahnestock y Secor, 1982: 273):

a) Las evaluaciones, argumentos de juicios de valor, son esencialmente argumentos de proposiciones categóricas (los juicios de valor situados en el predicado) cuando se mide el tema objeto de evaluación con una definición ideal de lo que ello debería ser, es decir, un criterio de perfección. Un argumento causal apoya a una evaluación cuando el criterio o definición ideal con la que medimos el objeto de evaluación incluye unas consecuencias que pueden ser buenas o malas.

b) Las propuestas utilizan proposiciones causales orientadas a la acción, hacia algo que debería, necesitaría hacerse. Aunque las consecuencias pueden ser, o no, relativamente obvias. Son tipos de argumentos muy comunes.

Con todo, la dinámica de un texto argumentativo es hacer pasar de un estado de pensamiento inicial (tesis rehusada) a un estado de pensamiento final (tesis propuesta) por medio de un proceso de argumentación ${ }^{38}$ (Boissinot, 1992: 37). Y, aunque el orden en la realización textual de este esquema, o modelo subyacente, puede diferir, el texto argumentativo desarrolla una dinámica, permitiendo pasar de una tesis a otra, y organiza un tipo de circuito argumentativo compuesto de un determinado número de argumentos. Entendiendo que el texto argumentativo no es solo y esencialmente el lugar de

36 «We have said that we do not argue about easily verified statements; we call them facts.» (Ibíd.:259).

37 «The only way to argue for something in the future is with a causal argument.» (Ibíd.:262).

${ }^{38}$ Igual que el texto narrativo hace pasar de un estado inicial a un estado final mediante un proceso de transformación (Boissinot, 1992: 37). 
un discurso contradictorio sobre la realidad, sino el discurso de diferentes posiciones sobre la realidad que pueden ser o no contradictorias. Estas diferentes posiciones posibilita la diversidad de argumentos que se pueden entretejer en un texto:

Argument, like language, o art, or ritual, is universal; but, again like language or art or ritual, it has many forms (Feyerabend ${ }^{39}$, 1987; en Leith y Myerson, 1989: 101).

En cuanto a las formas o modos de un argumento, Gilbert (1997) expone cuatro modos: el lógico (enfatiza el razonamiento silogístico o cuasi-lógico), el emocional (se apoya sobre el uso o expresión de la emoción), el visceral (considera la expresión física ${ }^{40}$, el lenguaje del cuerpo, y los recursos no verbales) y el kisceral (considera el rol de la intuición, el sentimiento religioso, y el misticismo como apoyo para los argumentos). Pero para Gilbert, el término clave es «coalescent argumentation ${ }^{41}$, refiriéndose a un conjunto de prácticas cuyo propósito es lograr el acuerdo entre los dos argumentadores, basado en la conjunción de sus posiciones de tantas maneras como sea posible.

Entendemos que en esta conjunción de posiciones, las expresiones lingüísticas empaquetan la experiencia que tenemos del mundo y codifican los diferentes puntos de vista que tenemos de ella, enmarcándose así en una determinada ideología (Fowler, 1996: 34). Así, la construcción de la realidad a través de la lengua no es neutral: ésta construye unos puntos de vista e ideologías en una situación discursiva y de diálogo. De modo que, siempre hay unos significados alternativos en los escenarios sociales del sistema semántico. Y la lengua ofrece a los interlocutores la posibilidad de intervención y de negociación en estos significados alternativos y escenarios sociales.

39 «Paul Feyerabend is a contemporary philosopher of science who currently exploits ideas of argument that are Rhetorical. He has been concerned to defend 'diversity': diverse views and diverse cultures especially. He suggests that the modern West threatens to impose 'increasing uniformity in the world'. Included in this uniformity is the suppression of argument — by dogmatic reason which knows all the answers» (Leith y Myerson, 1989: 101).

${ }^{40}$ Lo Cascio (1998: 114) plantea que la mirada amenazadora y airada de uno de los interlocutores puede considerarse como un argumento, aunque ello resulte discutible.

${ }^{41}$ Coalescent Argumentation es una argumentación centrada en el acuerdo. Se basa en que los argumentos son actividades sociales complejas que implican al ego humano buscar satisfacer sus necesidades intelectuales, emocionales, espirituales y físicas. En el transcurso de esta búsqueda hay desacuerdo y conflicto entre los individuos. Y éstos, siendo conscientes de ello, a través de la argumentación, pueden centrarse en asegurar sus necesidades e intentar satisfacer las de sus oponentes. 
Por consiguiente, la vida social es argumentativa (Antaki, 1994: 160). Y la producción de un texto argumentativo implica que es susceptible de ser discutido (Golder, 1996: 101), es decir, negociado.

\section{CONCLUSIONES}

La argumentación es principalmente una construcción o estructuración lingüística (que conlleva o indica un valor argumentativo, pero que también construye y estructura los enunciados). A la cual va unida el proceso linguístico-cognitivo de la inferencia, realizado por medio de la lengua, pero fundamentado en una determinada construcción de la realidad. Pero además, la argumentación se caracteriza por unas razones o significaciones que apoyan una afirmación o una tesis. Es decir, por unos tipos de argumentos que el discurso va avanzando, en el texto, de acuerdo con el pensamiento e interacción de los interlocutores, y de acuerdo con la naturaleza y el encadenamiento de las estructuras. Un argumento es un acto retórico, y su fuerza viene dada por la dimensión retórica de su acto de argumentar.

En esta construcción y estructuración lingüística, la argumentación, o acto de argumentar, puede ser tratada como una macro-argumentación discursiva y una micro-argumentación textual, habiendo entre ambas una relación de interdependencia y correspondencia, en consonancia con el carácter explícito e implícito de sus premisas, y de acuerdo con la situación retórica en la que se ubican. Aquí, la relación entre macro-argumentos y micro-argumentos es la relación entre lo explícito y lo implícito. Una relación que define a la argumentación como discurso (proceso) y como texto (producto). Con el matiz diferenciador de que las premisas explícitas se plasman en el producto (en el texto) y las premisas implícitas están situadas en el marco de su discurso. Conduciendo todo ello a un proceso ostensivo-inferencial determinado.

\section{REFERENCIAS BIBLIOGRÁFICAS}

Anscombre, J. C. y Ducrot, O. (1988). L'Argumentation dans la Langue. Liège: Pierre Mardaga Éditeur, 2. ${ }^{\mathrm{a}}$ ed $^{\mathrm{o}}$

AnTAKI, C. (1994). Explaining and Arguing. The Social Organization of Accounts. London: SAGE Publications.

Beaugrande, R. DE (1997). «The Story of Discourse Analysis». En Discourse as Structure and Process. Discourse Studies: A Multidisciplinary 
Introduction, T. A. van Dijk (ed.), vol. 1, 35-62. London: SAGE Publications.

BEAugrande, R. DE y DRESSLER, W. (1981). Introduction to Text Linguistics. London. New York: Longman.

Boissinot, A. (1992). Les Textes Argumentatifs. Toulouse: Bertrand-Lacoste.

Caron, J. (1992). An Introduction to Psycholinguistics. New Cork. London: Harvester Wheatsheaf.

CARrillo Guerrero, L. (2005). «Realización retórica del proceso discursivo y del producto textual». Odisea: Revista de Estudios Ingleses (Universidad de Almería) 6, 55-74.

Cox, J. R. y Willard, C. A. (1982). «Introduction. The Field of Argumentation». En Advances in Argumentation Theory and Research, J. R. Cox y C. A. Willard (eds.), xii-xlvii. Carbondale and Edwardsville, Southern Illinois University Press.

DiJK, T. A. VAN (1977). Text and Context. Explorations in the Semantics and Pragmatics of Discourse. London, New York: Longman.

- (1980). Macrostructures. An interdisciplinary study of global structures in discourse, interaction and cognition. Hillsdale: Lawrence Erlbaum Associates.

DiJK, T. A. VAN y KINTSCH, W. (1983). Strategies of Discourse Comprehension. New York: Academic Press.

DIK, S. C. (1997). The theory of Functional Grammar. [TFG] Ed. por Kees Hengeveld. Part 1, The Structure of the Clause (2. ${ }^{\mathrm{a}}$ ed. ${ }^{\circ}$ rev.) [TFG1]; Part 2, Complex and Derived Constructions. [TFG2]. Berlin: Mouton de Gruyter.

EDwards, D. (1997). Discourse and Cognition. London: SAGE Publications.

EEMEREn, F. van (2002). Argumentation: an Overview of Theoretical Approaches and Research Themes. University of Amsterdam.

http://www.argumentation.spb.ru/2002_1/papers/1_2002p4.html

- (2003). «The Development of the Pragma-dialectical Approach to Argumentation». Argumentation 17 (4), 387-403.

EEMEREn, F. H. van y GROOTENDORST, R. (1984). Speech Acts in Argumentative Discussions. A Theoretical Model for the Analysis of Discussions 
Directed towards Solving Conflicts of Opinion. Dordrechts: Foris Publications.

- (1987). Handbook of Argumentation Theory. Dordrechts: Foris Publications.

- (1988). «Rationale for a Pragma-Dialectical Perspective». Argumentation 2, 271-291.

- (1992). Argumentation, Communication, and Fallacies: a Pragma-Dialectical Perspective. Hillsdale, NJ.: Lawrence Erlbaum.

EEMEREN, F. H. van, GRoOTENDORSt, R. y KrUiger, T. (1984). The Study of Argumentation. New York: Irvington.

- (1987). Handbook of Argumentation Theory. Dordrecht: Foris Publications (Re-edición de Eemeren et al. 1984).

EEMEREN, F. H. van, GroOtendorst, R., JACKSON, S. y JACOBS, S. (1993). Reconstructing Argumentative Discourse. Tuscaloosa, AL - London: University of Alabama Press.

- (1997). «Argumentation». En Discourse as Structure and Process. Discourse Studies: A Multidisciplinary Introduction Volume 1, T. A. van Dijk (ed.), 208-229. London: SAGE Publications.

Eemeren, F. H. van, Grootendorst, R. y Snoeck H. F. (1996). Fundamentals of Argumentation Theory: A Handbook of Historical Background and Contemporary Developments. Mahwah, N.J.: Lawrence Erlbaum.

Eemeren, F. H. van y Houtlosser, P. (2000). «Rhetoric in Pragma-dialectics». Argumentation, Interpretation, Rhetoric 1, 1.

http://www.argumentation.spb.ru/2000_1/papers/1_2000p1.htm

EHNINGER, D. (1970). «Argument as Method: its Nature, its Limits and its Uses». Speech Monographs 37.

FAHNEStock, J. y SeCOR, M. (1982). A Rhetoric of Argument. New York: Random House.

FEYeRABEND, P. (1987). Farewell to Reason. London: Verso.

Fodor, J. A. (1979). The Language of Thought. Cambridge, Mass.: Harvard University Press.

FowleR, R. (1996) ( $2^{\text {nd }}$ edition $/ 1 .^{\text {a }}$ ed. $\left.{ }^{\circ} 1986\right)$. Linguistic Criticism. Oxford/New York: Oxford University Press. 
Gilbert, M. (1997). Coalescent Argumentation. New Jersey: Lawrence Erlbaum Associates.

GIvón, T. (1995). Functionalism and Grammar. Amsterdam, Philadelphia: John Benjamins.

Golder, C. (1996). Le Développement des Discours Argumentatifs. Lausanne: Delachaux et Niestlé.

Govier, T. A. (2001) (5. ${ }^{\mathrm{a}}$ ed. $\left.{ }^{\circ}\right)$. A Practical Study of Argument. Belmont, CA.: Wadsworth.

HABermas, J. (1981). Theorie des kommunikativen Handlens. Band 1. Handlungsrationalitat und gesellschaftliche Rationallalisierung. Frankfurt am Main: Suhrkamp Verlag. (Traducción española, Teoría de la acción comunicativa. I. Racionalidad de la acción y racionalidad social. Madrid: Taurus, 1987).

Halliday, M. A. K. (1978). Language as a Social Semiotic. The Social Interpretation of Language and Meaning. London: Edward Arnold.

JACKSON, S. y JACOBS, S. (1980). «Structure of Conversational Argument: Pragmatic Bases for the Enthymeme». Quarterly Journal of Speech 66, 251-65.

JACOBS, S. (1987). «The Management of Disagreement in Conversation». En Argumentation: Across the Line of Discipline 3, F. van Eemeren, R. Grootendorst, A. Balir, C. A. Willard (eds.), 229-239. Dordrecht: Foris Publication.

JACOBS, S. y JACKSON, S. (1982). «Conversational Argument: A Discourse Analytical Approach». En Advances in Argumentation Theory and Research, J. R. Cox y C. A. Willard (eds.), 205-237. Carbondale and Edwardsville, Ill.: Southern Illinois University Press.

- (1989). «Building a Model of Conversational Argument». En Rethinking communication, vol. 2, B. Dervin, L. Grossberg, B. O'Keefe y E. Wartella (eds.), 153-171. Newbury Park, CA., etc.: SAGE Publications.

KINTSCH, W. y DiJK, T. A. van (1978). «Towards a Model of Text Comprehension and Text Production». Psychological Review 85, 363-395.

Leith, D. y Myerson, G. (1989). The Power of Address. Explorations in rhetoric. London, New York: Routledge.

Lo CAscio, V. (1998). Gramática de la argumentación. Madrid: Alianza Editorial. (Versión española de David Casacuberta de: Grammatica 
dell' argomentare. Strategie e strutture. Scandicci (Firenze): La Nuova Italia Editrice, 1991).

MARTíneZ-DueÑAS, J. L. (2002). Retórica de la lengua inglesa. Granada: Comares.

MoesChler, J. (1985). Argumentation et conversation. Eléments pour une analyse pragmatique du discours. Paris: Hatier-Credif.

O’KeEFe, B. J. y Benort, P. J. (1982). «Children's Arguments». En Advances in Argumentation Theory \&Research, J. R. Cox y C. A. Willard (eds.), 154-183. Carbondale and Edwardsville, Ill.: Southern Illinois University Press.

Perelman, C. y Olbrechts-Tyteca, L. (1989). Tratado de la argumentación. La nueva retórica. Madrid: Gredos (traducción de Julia Sevilla de Traité de l'árgumentation. La nouvelle rhetorique, Bruxelles : Editions de l’Université de Bruxelles, 1976).

Rowland, R. (1987). «On Defining Argument». Philosophy and Rhetoric 20 (3), 140-159.

SCHIFFRIN, D. (1994). Approaches to Discourse. Oxford/Cambridge, Mass.: Basil Blackwell.

Toulmin, S. (1958). The Uses of Argument. Cambridge: Cambridge University Press, 2. ${ }^{\text {a }}$ ed. ${ }^{\circ}$ 1969; reimpreso, 1986.

Toulmin, S., RIEKE, R. y JANIK, A. (1984). An Introduction to Reasoning. New York: Macmillan, 2. ${ }^{\mathrm{a}}$ ed. ${ }^{\circ}$.

VignauX, G. (1988). Le Discours acteur du monde. Enonciation, argumentation et cognition. Paris: Ophyrs.

Walton, D. N. (1989). Informal Logic: a Handbook for Critical Argumentation. Cambridge: Cambridge University Press.

- (1995). A Pragmatic Theory of Fallacy. Tuscaloosa, AL.: The University of Alabama Press.

Willard, C. A. (1983). Argumentation and the Social Grounds of Knowledge. Tuscaloosa AL.: The University of Alabama Press.

- (1989). A Theory of Argumentation. Tuscaloosa, AL - London: The University of Alabama Press. 